JGP - Vol. 6, n. 2, 1989

\title{
Hamiltonian formulation of adiabatic free boundary Euler flows
}

\author{
ARTHUR MAZER, TUDOR RATIU \\ Department of Mathematics \\ University of Arizona, Tucson, AZ 85721 \\ Department of Mathematics \\ University of California, Santa Cruz, CA 95064
}

\begin{abstract}
A Hamiltonian formulation of adiabatic free boundary inviscid fluid flow using only physical variables is presented in both the material and spatial formulation. Using the symmetry of particle relabeling, we derive the noncanonical Poisson bracket in Eulerian representation as a reduction from the canonical bracket in Lagrangian representation. When the free boundary of the fluid is given as the zero set of a function dragged along by the fuid fow, there is another bracket due to Abarbanel et al. [Physics of Fluids, (vol. 31), (2802), (1988)]. It is shown that this formulation «covers» the present one by proving that the natural restriction map is Poisson. It is also shown that the potential vortycity and the conserved quantities found by Abarbanel and Holm [Physics of Fluids (vol. 30), (3369), (1987)] are also conserved in the free boundary case.
\end{abstract}

\section{INTRODUCTION}

This paper extends previous results of Lewis et al. [1] to the case of ideal adiabatic self-gravitating flow with surface tension, obtaining a Hamiltonian formulation of this problem. This is a step in a larger program concerned with the stability and bifurcation analysis of fluid equilibria. For the case of a fluid with surface tension such a program was carried out in Lewis et al. [2] and Lewis [3]. Other relevant problems would be the study of weather systems, the free boundary being the outer surface of the atmosphere, or oceanographic problems where the water surface is the free boundary. Irrotational free

Key-Words: Hamiltonian formulation, Euler flows 1980 MSC: 76 B 99 
surface problems have been studied from a Hamiltonian point of view by Zakharov [4], Broer [5], Miles [6], Milder [7], Benjamin and Olver [8], and Benjamin [8] and our work naturally extends these results. There are also canonical Hamiltonian formulations of rotational free boundary value flow but these use non-physical variables. See Henyey and Wright [9] for an attempt at combining these two approaches and Marsden et al. [10] and references therein for a modern approach, involving momentum maps, that explains how such non-physical variables as «Cayley-Klein parameters» and various «Clebsch variables» can be obtained in a systematic manner. The main purpose of this paper is on the one hand to have only physical variables in the non-canonical Poisson bracket and on the other hand not just to exhibit but to understand its derivation from a geometric point of view.

The by now standard method used to obtain the Poisson bracket is the reduction from Lagrangian to Eulerian representation by climinating the gauge symmetry of particle relabeling. The main complication is the free boundary $\Sigma$ which we take to be an unparameterized surface in $\mathbb{R}^{3}$. Its equation of motion simply states that at every point of $\Sigma$, the time derivative equals the normal component of the velocity, i.e., $\partial \sigma / \partial t=$ $\mathbf{v} \cdot \nu$ where $\nu$ is the outward unit normal to $\Sigma$. We think therefore of variations of $\Sigma$ as functions on $\Sigma$. The structure of the bracket is reminiscent of the one for the fixed boundary case to which one adds boundary correction terms, one of them as in Lewis et al. [1].

The content of the paper is as follows. Sec. 2 presents the Lagrangian framework. The Poisson bracket for the Eulerian description is derived via reduction in section 3. In section 4 , we explicitly calculate the functional derivatives of a class of functions. Similar computations were done for the self-gravitating potential, by hand, by Poincaré [21]. These calculations are then used in section 5 where the equations of motion for an ideal adiabatic self gravitating flow with surface tension are deduced from the bracket. The equations of motion are the Eulcr equations over a domain with a frec boundary, supplemented by an equation of motion for the boundary. This example explicity demonstrates that the equations are Hamiltonian. In section 4, we show how to pass between the formulation of Abarbanel et al. [11] and the formulation presented here.

\section{THE POISSON BRACKET IN LAGRANGIAN REPRESENTATION}

The configuration space of a fluid in Lagrangian representation is a collection of mappings $\eta$ from a space $D$ of fluid labels to the locations in physical spacc $\mathbb{R}^{3}$, of fluid particles: $\eta: D \rightarrow \mathbb{R}^{3}$. The three-dimensional region $D$ is assumed to have a smooth boundary $\partial D$ and its interior to equal $D$ minus its boundary. We shall assume that $\eta$ maps the interior of $D$ to the interior of the fluid and $\partial D$ to the boundary of the fluid (so we take $\eta$ to be an cmbedding). Thus the configuration space consists of the collection of these $\eta$ 's, i.e., it equals $\mathbf{C}=\operatorname{Emb}\left(D, \mathbb{R}^{3}\right)$, the manifold of cmbeddings 
of $D$ into $\mathbf{R}^{3}$. To each point value in the label space, $\mathbf{X} \in D$, we associate the mass per label volume $\rho_{0}(\mathbf{X})$ and the specific entropy $\sigma_{0}(\mathbf{X})$ of labeled fluid element. The measure of mass $\rho_{0}(\mathbf{X}) \mathrm{d}^{3} \mathbf{X}$ will be callcd the material mass density of the fluid and the function $\sigma_{0}(\mathbf{X})$, the material entropy of the fluid. A point in phase space consists of a configuration $\eta$ and a momentum density $\mu=\mu^{\prime} \otimes \mathrm{d}^{3} \mathbf{X}$, where $\mu^{\prime}(\mathbf{X}$ is the momentum per label volume, i.e., the material phase space of the fluid is the cotangent space $T^{*} \mathbf{C} \times\left\{\left(\rho_{0}, \sigma_{0}\right)\right\}$ which we now describe.

The tangent space $T_{\eta} \mathbf{C}$ to $\mathbf{C}$ at $\eta$ consists of maps $\delta \eta: D \rightarrow \mathbf{R}^{3}$ such that $\delta \eta(\mathbf{X})$ is a tangent vector to $\mathbf{R}^{3}$ at $\eta(\mathbf{X})$. Therefore, the cotangent space $T_{\eta}^{*} \mathbf{C}$ consists oneform densities $\mu$ over $\eta$, i.e., $\mu=\mu^{\prime} \otimes \mathrm{d}^{3} \mathbf{X}$ such that $\mu^{\prime}(\mathbf{X})$ is a one-form on $\mathbf{R}^{3}$ at $\eta(\mathbf{X}) ; \mu \in T^{*} \mathbf{C}$ acts on $T \mathbf{C}$ by the usual $L^{2}$-pairing

$$
\langle\mu, \delta \eta\rangle=\int_{D} \mu^{\prime}(\mathbf{X}) \cdot \delta \eta(\mathbf{X}) \mathrm{d}^{3} \mathbf{X},
$$

where $\mu^{\prime}(\mathbf{X}) \cdot \delta \eta(\mathbf{X})$ denotes the usual contraction of a one-form at $\eta(\mathbf{X})$ with a vector at $\eta(\mathbf{X})$.

If $\eta_{t}(\mathbf{X})$ denotes the path traced out by the particle labeled by $\mathbf{X} \in D$ ( $D$ is also called the reference space), and $d^{3} \mathbf{x}$ is the usual volume form on $\mathbf{R}^{3}$, then the Eulerian fluid density $\rho$ and the Eulerian entropy $\sigma$ are given by

$$
\eta_{t}^{*}\left(\rho \mathrm{d}^{3} \mathrm{x}\right)=\rho_{0} \mathrm{~d}^{3} \mathbf{X}, \quad \eta_{t}^{*} \sigma=\sigma_{0}
$$

or equivalently by

$$
\left(\rho \circ \eta_{t}\right) J\left(\eta_{t}\right)=\rho_{0} \quad \text { and } \quad \sigma \circ \eta_{t}=\sigma_{0}
$$

where $J\left(\eta_{t}\right)(\mathbf{X})=\mathrm{d}^{3} \mathbf{x} / \mathrm{d}^{3} \mathbf{X}$ is the Jacobian determinant of $\eta_{t}$ and $\mathbf{x}$ is the usual fixed spatial observation position for the Eulerian description of fluid dynamics. Taking the time derivative of (2) or (3) gives the differential version of the mass and entropy conservation

$$
\frac{\partial \rho}{\partial t}+\operatorname{div}(\rho \mathbf{v})=0, \quad \frac{\partial \sigma}{\partial t}+\mathbf{v} \cdot \nabla \sigma=0
$$

where $\mathbf{v}(t, \mathbf{x})=\partial \eta_{t}(\mathbf{X}) / \partial t$, i.e., $\mathbf{v} \circ \eta_{t}=\mathbf{V}$.

The thermodynamic variables $\rho(\mathbf{x}, t)$ and $\sigma(\mathbf{x}, t)$ enter the dynamics of the fluid through the fluid pressure $p(\rho, \sigma)$ or equivalently the energy density $e(\rho, \sigma): p=$ $\rho^{2} \partial e / \partial p$.

Let $\mathbf{F}$ denote the functions on $D, \mathbf{F}^{*}$ the densities on $D$, and let

$$
\left\langle f \mathrm{~d}^{3} \mathbf{X}, g\right\rangle=\int_{D} f(\mathbf{X}) g(\mathbf{X}) \mathrm{d}^{3} \mathbf{X}
$$


be the usual $L^{2}$-pairing between $\mathbf{F}$ and $\mathbf{F}^{*}$. Since the phase space of the fluid is the symplectic manifold $T^{*} \mathbf{C} \times\left\{\left(\rho_{0}, \sigma_{0}\right)\right\}$, the phase space of the collection of all adiabatic fluids is the Poisson manifold $T^{*} \mathbf{C} \times \mathbf{F}^{*} \times \mathbf{F}$ where $T^{*} \mathbf{C}$ caries the canonical sympletic structure and $\mathrm{F}^{*} \times \mathrm{F}$ the trivial bracket.

The Poisson bracket on $T^{*} \mathbf{C}$ is canonical. To write it out explicitly we need to define the partial functional derivatives of $F: T^{*} \mathbf{C} \rightarrow \mathbf{R}$. This needs to be done carefully in order to capture the boundary terms. The partial Fréchet derivative of $F$ relative to $\mu$ is defined by

$$
\mathbf{D}_{\mu} F(\eta, \mu) \cdot \delta \mu=\left.\frac{\mathrm{d}}{\mathrm{d} \varepsilon}\right|_{\varepsilon=0} F(\eta, \mu+\varepsilon \delta \mu)
$$

It is no easy to define the partial Fréchet derivative relative relative to $\eta$. We proceed as in Lewis et al. [1]. Identify $T^{*}(\eta(D))$ with $\left.\eta(D) \times \mathbf{R}^{3}\right)^{*}$ and let $\tilde{\mu}$ be the projection of $\mu / \mathrm{d}^{3} \mathbf{X}$ onto $\left(\mathbf{R}^{3}\right)^{*}$, so that $\mu / \mathrm{d}^{3} \mathbf{X}=\eta \times \tilde{\mu}: D \rightarrow \eta(D) \times\left(\mathbb{R}^{3}\right)^{*}=$ $T^{*}(\eta(D))$. Given a tangent vector $\delta \eta \in T_{\eta} \mathbf{C}$ and a curve $\eta_{\varepsilon}$ tangent to $\delta \eta$ at $\varepsilon=0, \eta_{0}=\eta$, let $\mu_{\varepsilon}=\left(\eta_{\varepsilon} \times \tilde{\mu}\right) \mathrm{d}^{3} \mathbf{X}$ and define

$$
\mathbf{D}_{\eta} F(\eta, \mu) \cdot \delta \eta=\left.\frac{\mathrm{d}}{\mathrm{d} \varepsilon}\right|_{\varepsilon=0} F\left(\eta_{\varepsilon}, \mu_{\varepsilon}\right) .
$$

Now we are ready to define the partial functional derivatives of $F: T^{*} \mathbf{C} \rightarrow \mathbb{R}$ as

$$
\begin{aligned}
& \frac{\delta F}{\delta \eta}=\frac{\delta^{\wedge} F}{\delta \eta}+\delta_{\partial D} \frac{\delta^{\sim} F}{\delta \eta} \\
& \frac{\delta F}{\delta \mu}=\frac{\delta^{\wedge} F}{\delta \mu}+\delta_{\partial D} \frac{\delta^{\sim} F}{\delta \mu}
\end{aligned}
$$

where $\delta_{\partial D}$ is the Dirac delta function of the boundary $\partial D$ and

(i) $\delta^{\wedge} F / \delta \eta$ is a one-form density over $\eta$ on $D$,

(ii) $\delta \sim F / \delta \eta$ is a one-form density over $\eta$ on $\partial D$,

(iii) $\delta^{\wedge} F / \delta \mu$ is a vector field over $\eta$ on $D$,

(iv) $\delta \breve{F} / \delta \mu$ is a vector ficld over $\eta$ on $\partial D$, such that

$$
\begin{aligned}
& \mathbf{D}_{\mu} F(\eta, \mu) \cdot \delta \eta=\int_{D} \frac{\delta^{\wedge} F}{\delta \eta} \cdot \delta \eta+\int_{D} \frac{\delta^{\sim} F}{\delta \eta} \cdot \delta \eta \\
& \mathbf{D}_{\mu} F(\eta, \mu) \cdot \delta \mu=\int_{D} \delta \mu \cdot \frac{\delta^{\wedge} F}{\delta \mu}+\delta \mu \cdot \frac{\delta^{\sim} F}{\delta \mu}
\end{aligned}
$$

for all variations $\delta \eta \in T C, \delta \mu=$ a one-form density on $D$ over $\eta$. The canonical Poisson bracket on $T^{*} \mathrm{C}$ is given for any two functions $F, K: T^{*} \mathbf{C} \rightarrow \mathbf{R}$ by

$$
\{\{F, K\}\}(\eta, \mu)=\int_{D}\left(\frac{\delta F}{\delta \eta} \cdot \frac{\delta K}{\delta \mu}-\frac{\delta K}{\delta \eta} \cdot \frac{\delta F}{\delta \mu}\right) .
$$


In order for (12) to be well defined, in view of (8) and (9) we need to require that the functions $F, K$ we consider satisfy

$$
\frac{\delta^{\sim} F}{\delta \eta} \cdot \frac{\delta^{\sim} K}{\delta \mu}-\frac{\delta^{\sim} K}{\delta \eta} \cdot \frac{\delta^{\sim} F}{\delta \mu}=0 .
$$

(Squares of delta functions need to be avoided). For example, we could work in the space of functionals $F$ for which $\delta^{\triangleleft} F / \delta \mu$ or $\delta^{`} F / \delta \eta$ vanish. However, rather than restricting the class of functionals we shall simply assume that (13) holds in the sequel. For such functions the canonical Poisson bracket becomes

$$
\begin{aligned}
& \{\{F, G\}\}=\int_{D}\left(\frac{\delta^{\sim} F}{\delta \eta} \cdot \frac{\delta^{\sim} K}{\delta \mu}-\frac{\delta^{\curvearrowright} K}{\delta \eta} \cdot \frac{\delta^{\searrow} F}{\delta \mu}\right)+ \\
& +\int_{D}\left(\frac{\delta^{\wedge} F}{\delta \eta} \cdot \frac{\delta^{`} K}{\delta \mu}-\frac{\delta^{\wedge} K}{\delta \eta} \cdot \frac{\delta^{\curvearrowright} F}{\delta \mu}\right)+ \\
& +\int_{D}\left(\frac{\delta^{\sim} F}{\delta \eta} \cdot \frac{\delta^{\wedge} K}{\delta \mu}-\frac{\delta^{\sim} K}{\delta \eta} \cdot \frac{\delta^{\wedge} F}{\delta \mu}\right) \text {. }
\end{aligned}
$$

\section{DERIVATION OF THE BRACKET BY REDUCTION FROM THE LAGRAN- GIAN REPRESENTATION}

The dynamical variables we need for the Eulerian description of the fluid are the free boundary $\Sigma, \rho$ and $\sigma$ as introduced earlier, and the Eulerian velocity $\mathbf{v}(\mathbf{x}, t)$. We shall work with the momentum density $\mathbf{M}=\rho \mathbf{v}$ rather than $\mathbf{v}$ itself. We want to translate the information we have on the fields, $\eta$ and $\mu$ in Lagrangian representation to the Eulerian fields $\Sigma, \rho, \sigma$, and $\mathbf{M}$ and find a closed set of Poisson brackets among the latter. It is reduction that allows us to do just that.

There are two equivalent ways to reduce: to consider the family of all possible fluids (i.e., to let $\rho_{0}, \sigma_{0}$ be variables) and to divide by the semidirect product group of the diffeomorphisms of $D$ with the linear space of functions and densities on $D$, or to fix $\rho_{0}$ and $\sigma_{0}$, Poisson reduce by the particle relabeling group defined by $\rho_{0}$ and $\sigma_{0}$, and then take the union of the resulting manifolds, thereby making the total mass and total entropy Casimirs. In the first approach, this latter statement is a direct consequence of the geometric construction. The resulting manifolds are in the first approach $\mathbf{E}^{\prime}=$ $\left\{(\Sigma, \mathbf{M}, \rho, \sigma) \mid \rho=\left(\rho_{0} \circ \phi^{-1}\right)\left(J(\phi)^{-1} \circ \phi^{-1}\right), \sigma=\sigma_{0} \circ \phi^{-1}\right.$, for some $\rho_{0}, \sigma_{0}$, and $\phi \in \mathbf{C}\}$ and in the second $\mathbf{E}=\left\{(\Sigma, \mathbf{M}, \rho, \sigma) \mid \rho=\left(\rho_{0} \circ \phi^{-1}\right)\left(J(\phi)^{-1} \circ \phi^{-1}\right)\right.$, $\sigma=\sigma_{0} \circ \phi^{-1}$, for some $\left.\phi \in \mathbf{C}\right\}$. Thus $\mathbf{E}$ depends on $\left(\rho_{0}, \sigma_{0}\right)$ and the union of all these $\mathbf{E}$ is $\mathbf{E}^{\prime}$. Each $\mathbf{E}$ is a Poisson submanifold of the physical manifold $\mathbf{E}^{\prime}$. The theory for the first approach is developed in Holm, Marsden, and Ratiu [20], where its equivalence with the second method is proved in a very general geometric context. We shall not proceed here along these lines since it is technically involved and brings in se- 
midirect product theory in a non-trivial fashion. Rather, we shall fix $\rho_{0}$ and $\sigma_{0}$, Poisson reduce from the material representation and simply observe at the end that the union of all the manifolds $\mathbf{E}$ over $\rho_{0}$ and $\sigma_{0}$ form a new manifold $\mathbf{E}^{\prime}$ on which the derived bracket makes sense, by simply declaring each $\mathbf{E}$ to be a Poisson submanifold of $\mathbf{E}^{\prime}$.

Now we consider the manifold $\mathbf{E}$ of Eulerian fields $(\Sigma, \mathbf{M}, \rho, \sigma)$ such that

$$
\rho=\left(\rho_{0} \circ \phi^{-1}\right)\left(J(\phi)^{-1} \circ \phi^{-1}\right) \text { and } \sigma=\sigma_{0} \circ \phi^{-1}
$$

for some $\phi \in \mathbf{C} ; \rho_{0}$ and $\sigma_{0}$ are fixed throughout the ensuing discussion. If $F$ is a functional on $\mathbf{E}$, define the functional derivatives evaluated at a point in $\mathbf{E}$ as follows. Let $\left(\frac{\delta F}{\delta \Sigma}, \frac{\delta F}{\delta \mathbf{M}}, \frac{\delta F}{\delta \rho}, \frac{\delta F}{\delta \sigma}\right)$ be the unique element of the tangent space $T_{(\Sigma, \mathbf{M}, \rho, \sigma)} \mathbf{E}$ satisfying

$$
\begin{aligned}
\mathbf{d} F(\Sigma, \mathbf{M}, \rho, \sigma) & \cdot(\delta \Sigma, \delta \mathbf{M}, \delta \rho, \delta \sigma)= \\
& =\int_{\Sigma} \frac{\delta F}{\delta \Sigma} \delta \Sigma \mathrm{d} A+\int_{D_{\Sigma}}\left(\frac{\delta F}{\delta \mathbf{M}} \delta \mathbf{M}+\frac{\delta F}{\delta \rho} \delta \rho+\frac{\delta F}{\delta \sigma} \delta \sigma\right) \mathrm{d}^{3} \mathbf{x}
\end{aligned}
$$

for all $(\delta \Sigma, \delta \mathbf{M}, \delta \rho, \delta \sigma) \in T_{(\Sigma, \mathbf{M}, \rho, \sigma)} \mathbf{E}$ where $\mathbf{d}$ is the differential on $\mathbf{E}, \mathrm{d} A$ denotes the measure on $\Sigma$ induced from the usual Lebesgue measure $\mathrm{d}^{3} \mathbf{x}$ on $\mathbb{R}^{3}, D_{\Sigma}$ is the region enclosed by $\Sigma$, and $T_{(\Sigma, \mathbf{M}, \rho, \sigma)} \mathbf{E}$ denotes the tangent space to the manifold $\mathbf{E}$ at $(\Sigma, \mathbf{M}, \rho, \sigma)$.

Define as in Lewis et al. [1] the map $\Pi: T^{*} \mathbf{C} \times\left\{\left(\rho_{0}, \sigma_{0}\right)\right\} \rightarrow \mathbf{E}$ by

$$
\Sigma=\partial(\eta(D)), \quad \rho=\left(\rho_{0} \circ \eta^{-1}\right)\left(J(\eta)^{-1} \circ \eta^{-1}\right), \quad \sigma=\sigma_{0} \circ \eta^{-1}
$$

and $\mathbf{M}$ is given as the unique vector field on $D_{\Sigma}$ such that for all other vector fields $\mathbf{w}$ on $\mathbb{R}^{3}$ we have

$$
\mathbf{M}(\mathbf{x}) \cdot \mathbf{w}(\mathbf{x})=\left\langle\mu(\mathbf{X}) / J(\eta)(\mathbf{X}) \mathrm{d}^{3} \mathbf{X},(\mathbf{w} \circ \eta)(\mathbf{X})\right\rangle
$$

where - on the left is the usual dot-product in $\mathbb{R}^{3},\langle$,$\rangle on the right is the contraction$ of a one-form with a vector field at $\mathbf{x}=\eta(\mathbf{X})$, and $\mu(\mathbf{X}) / J(\eta)(\mathbf{X}) \mathrm{d}^{3} \mathbf{X}$ denotes the one-form over $\eta$ which when tensored with $J(\eta)(\mathbf{X}) \mathrm{d}^{3} \mathbf{X}$ gives the one-form density $\mu(\mathbf{X})$. A relabcling of the fluid particles in Lagrangian representation which preserves $\rho_{0}(\mathbf{X})$ and $\sigma_{0}(\mathbf{X})$ will result in the same physics seen in Eulcrian representation. Relabeling forms a group, and wc want to look at the fluid orbits which are equivalent under this group. The group is $G=\left\{\phi\right.$ a diffeomorphism of $D \mid \sigma_{0} \circ \phi=\sigma_{0}$, $\left.\left(\rho_{0} \circ \phi\right) J(\phi)=\rho_{0}\right\}$. Under a right action of $G, \Pi$ is invariant, thus inducing a map of the quotient $G \backslash\left(T^{*} \mathbf{C} \times\left\{\left(\rho_{0}, \sigma_{0}\right)\right\}\right) \rightarrow \mathbf{E}$ which is easily checked to be a bijection. 
Given the right manifold of maps structure, $\Pi$ is a diffeomorphism. We shall induce a bracket $\{$,$\} on \mathbf{E}$ by the relation

$$
\{F, K\} \circ \Pi=\{\{F \circ \Pi, K \circ \Pi\}\} .
$$

Given $F: \mathbf{E} \rightarrow \mathbb{R}$, let $F^{\prime}=F \circ \Pi$. To compute $\mathbf{D}_{\mu} F$, let $\delta \mathbf{M}$ be given by

$$
\delta \mathbf{M}(\mathbf{x}) \cdot \mathbf{w}(\mathbf{x})=\left\langle\mu(\mathbf{M}) / J(\eta)(\mathbf{X}) \mathrm{d}^{3} \mathbf{X}, \quad(\mathbf{w} \circ \eta)(\mathbf{X})\right\rangle
$$

so that $\mathbf{M}+\varepsilon \delta \mathbf{M}$, with $\mathbf{M}$ given by (17), is the second component of $\Pi(\eta, \mu+\varepsilon \delta \mu)$. Thus by (6), (15), (19), and the change of variables $\mathbf{x}=\eta(\mathbf{X})$, we get

$$
\begin{aligned}
\mathbf{D}_{\mu} F^{\prime}(\eta, \mu) \cdot \delta \mu & =\left.\frac{\mathrm{d}}{\mathrm{d} \varepsilon}\right|_{\varepsilon=0} F^{\prime}(\eta, \mu+\varepsilon \delta \mu)= \\
& =\mathbf{D}_{\mathbf{M}} F(\Sigma, \mathbf{M}, \rho, \sigma) \cdot \delta \mathbf{M}=\int_{D_{\mathbf{x}}} \frac{\delta F}{\delta \mathbf{M}}(\mathbf{x}) \cdot \delta \mathbf{M}(\mathbf{x}) \mathrm{d}^{3} \mathbf{x}= \\
& =\int_{D}\left\langle\frac{\delta \mu(\mathbf{X})}{J(\eta)(\mathbf{X}) \mathrm{d}^{3} \mathbf{X}},\left(\frac{\delta F}{\delta \mathbf{M}} \circ \eta\right)(\mathbf{X})\right\rangle J(\eta)(\mathbf{X}) \mathrm{d}^{3} \mathbf{X}= \\
& =\int_{D} \delta \mu(\mathbf{X}) \cdot\left(\frac{\delta F}{\delta \mathbf{M}} \circ \eta\right)(\mathbf{X}),
\end{aligned}
$$

so that using definition (11), we get

$$
\frac{\delta^{\wedge} F^{\prime}}{\delta \mu}=\frac{\delta F}{\delta \mathbf{M}} \circ \eta, \quad \frac{\delta^{`} F^{\prime}}{\delta \mu}=0 .
$$

To compute the $\eta$-derivative, write as in section $3, \mu / \mathrm{d}^{3} \mathbf{X}=\eta \times \tilde{\mu}: D \rightarrow \eta(D) \times$ $\left(\mathbb{R}^{3}\right)^{*}=T^{*}(\eta(D))$, let $\delta \eta \in T_{\eta} \mathbf{C}$ be given, let $\eta_{\boldsymbol{\varepsilon}}$ be a curve which at $\varepsilon=0$ equals $\eta$ and whose tangent vector at $\varepsilon=0$ is $\delta \eta$, and denote $\mu_{\varepsilon}=\left(\eta_{\varepsilon} \times \tilde{\mu}\right) \mathrm{d}^{3} \mathbf{X}$. Let $\mathbf{x}=\eta(\mathbf{X})$ and denote

$$
\begin{aligned}
& \delta \Sigma(\mathbf{x})=\delta \eta(\mathbf{X}) \cdot \nu(\mathbf{x}), \quad \text { for } \quad \mathbf{X} \in \partial D, \mathbf{x} \in \Sigma \\
& \delta \sigma(\mathbf{x})=\left.\frac{\mathrm{d}}{\mathrm{d} \varepsilon}\right|_{\varepsilon=0}\left(\sigma_{0} \circ \eta_{\varepsilon}^{-1}\right)(\mathbf{x})=-\left\langle\mathbf{d} \sigma_{0} \circ \eta^{-1}, T \eta^{-1} \circ \delta \eta \circ \eta^{-1}\right\rangle(\mathbf{x})= \\
&=-\left\langle\mathrm{d}\left(\sigma_{0} \circ \eta^{-1}\right)(\mathbf{x}), \delta \eta(\mathbf{X})\right\rangle=-\nabla \sigma(\mathbf{x}) \cdot \delta \eta(\mathbf{X}), \\
& \delta \rho(\mathbf{x})=\left.\frac{\mathrm{d}}{\mathrm{d} \varepsilon}\right|_{\varepsilon=0}\left(\rho_{0} \circ \eta_{\varepsilon}^{-1}\right)\left(J\left(\eta_{\varepsilon}\right)^{-1} \circ \eta_{\varepsilon}^{-1}\right)(\mathbf{x})= \\
&=-\nabla\left(\rho_{0} \circ \eta^{-1}\right)(\mathbf{x}) \cdot \delta \eta(\mathbf{X}) J(\eta)(\mathbf{X})^{-1}- \\
&-\left.\left(\rho_{0} \circ \eta^{-1}\right)(\mathbf{x}) J(\eta)(\mathbf{X})^{-2} \frac{\mathrm{d}}{\mathrm{d} \varepsilon}\right|_{\varepsilon=0} J\left(\eta_{\varepsilon}\right)(\mathbf{X})- \\
&-\left(\rho_{0} \circ \eta^{-1}\right)(\mathbf{x}) \nabla\left(J(\eta)^{-1} \circ \eta^{-1}\right)(\mathbf{x}) \cdot \delta \eta(\mathbf{X})= \\
&=-\nabla \rho(\mathbf{x}) \cdot \delta \eta(\mathbf{X})-\left.\rho(\mathbf{x}) J(\eta)(\mathbf{X})^{-1} \frac{\mathrm{d}}{\mathrm{d} \varepsilon}\right|_{\varepsilon=0} J\left(\eta_{\varepsilon}\right)(\mathbf{X}) .
\end{aligned}
$$


To finish the computation of $\delta \rho$, we determine the $\varepsilon$-derivative of $J\left(\eta_{\varepsilon}\right)$ as follows. The relation $\eta_{\varepsilon}^{*} \mathrm{~d}^{3} \mathbf{x}=J\left(\eta_{\varepsilon}\right) \mathrm{d}^{3} \mathbf{X}$ implies that $\left(\eta_{\varepsilon} \circ \eta^{-1}\right)^{*} \mathrm{~d}^{3} \mathbf{x}=\left(J\left(\eta_{\varepsilon}\right) \circ \eta^{-1}\right)$ $J\left(\eta^{-1}\right) \mathrm{d}^{3} \mathbf{x}$ so that taking the $\varepsilon$-derivative at $\varepsilon=0$ yields $\operatorname{div}\left(\delta \eta \circ \eta^{-1}\right)=$ $\left.\frac{\mathrm{d}}{\mathrm{d} \varepsilon}\right|_{\varepsilon=0}\left(J\left(\eta_{\varepsilon}\right) \circ \eta^{-1}\right) J\left(\eta^{-1}\right)$, or

$$
J(\eta)(\mathbf{X}) \operatorname{div}\left(\delta \eta \circ \eta^{-1}\right)(\mathbf{x})=\left.\frac{\mathrm{d}}{\mathrm{d} \varepsilon}\right|_{\varepsilon=0} J\left(\eta_{\varepsilon}\right)(\mathbf{X})
$$

Therefore (23) becomes

$$
\begin{aligned}
\delta \rho(\mathbf{x}) & =-\nabla \rho(\mathbf{x}) \cdot \delta \eta(\mathbf{X})-\rho(\mathbf{x}) \operatorname{div} \delta \eta(\mathbf{X})= \\
& =-\operatorname{div}\left(\rho\left(\delta \eta \circ \eta^{-1}\right)\right)(\mathbf{x})
\end{aligned}
$$

Finally, writing $\mu_{\varepsilon}=\left(\eta_{\varepsilon} \times \tilde{\mu}\right) \mathrm{d}^{3} \mathbf{X}$ where $\tilde{\mu}$ is the projection of $\mu / \mathrm{d}^{3} \mathbf{X}$ to $\left(\mathbb{R}^{3}\right)^{*}$ in the trivialization $T^{*}(\eta(D))=\eta(D) \times\left(\mathbb{R}^{3}\right)^{*}$, (17) implies that

$$
\mathbf{M}(\mathbf{x})=J(\eta)(\mathbf{X})^{-1} \tilde{\mu}(\mathbf{X})
$$

so that by (24)

$$
\begin{aligned}
\delta \mathbf{M}(\mathbf{x})= & \left.\frac{\mathrm{d}}{\mathrm{d} \varepsilon}\right|_{\varepsilon=0}\left(J\left(\eta_{\varepsilon}\right) \circ \eta^{-1}\right)(\mathbf{x})^{-1}\left(\tilde{\mu} \circ \eta_{\varepsilon}^{-1}\right)(\mathbf{x})= \\
= & -\left.J(\eta)(\mathbf{X})^{-2} \frac{\mathrm{d}}{\mathrm{d} \varepsilon}\right|_{\varepsilon=0} J\left(\eta_{\varepsilon}\right)(\mathbf{X}) \tilde{\mu}(\mathbf{X})- \\
& -\left\langle\mathbf{d} J(\eta)(\mathbf{X})^{-1},\left(T \eta^{-1} \circ \delta \eta \circ \eta^{-1}\right)(\mathbf{x})\right\rangle \tilde{\mu}(\mathbf{X})- \\
& \left.-J(\eta)(\mathbf{X})^{-1}\left\langle\mathbf{d} \tilde{\mu}(\mathbf{X}), T \eta^{-1} \circ \delta \eta \circ \eta^{-1}\right)(\mathbf{x})\right\rangle= \\
= & -J(\eta)(\mathbf{X})^{-1} \tilde{\mu}(\mathbf{X}) \operatorname{div}\left(\delta \eta \circ \eta^{-1}\right)(\mathbf{x})- \\
& -\left\langle\mathbf{d}\left(J(\eta)^{-1} \circ \eta^{-1}\right)(\mathbf{x}),\left(\delta \eta \circ \eta^{-1}\right)(\mathbf{x})\right\rangle \tilde{\mu}(\mathbf{X})- \\
& -J(\eta)(\mathbf{X})^{-1}\left\langle\mathbf{d}\left(\tilde{\mu} \circ \eta^{-1}\right),\left(\delta \eta \circ \eta^{-1}\right)(\mathbf{x})\right\rangle= \\
= & -\mathbf{M}(\mathbf{x}) \operatorname{div}\left(\delta \eta \circ \eta^{-1}\right)(\mathbf{x})- \\
& -\left\langle\mathbf{d}\left(\left(J(\eta)^{-1} \circ \eta^{-1}\right)\left(\tilde{\mu} \circ \eta^{-1}\right)(\mathbf{x}),\left(\delta \eta \circ \eta^{-1}\right)(\mathbf{x})\right\rangle=\right. \\
= & -\mathbf{M}(\mathbf{x}) \operatorname{div}\left(\delta \eta \circ \eta^{-1}\right)(\mathbf{x})-\left(\left(\delta \eta \circ \eta^{-1}\right)(\mathbf{x}) \cdot \nabla\right) \mathbf{M}(\mathbf{x})
\end{aligned}
$$

Remark. $\delta \mathbf{M}$ in (26) represents only the variation of $\mathbf{M}$ due to a variation in $\eta$. Therefore the full variation of $\mathbf{M}$ equals the sum of the expression in (12) and $\delta \mu \circ \eta^{-1}$, 
which is the variation of $\mathbf{M}$ due to a variation in $\mu$. Thus, $\delta \mathbf{M}$ is an unrestricted vector field on $D_{\Sigma}$. From (21), (22), (25), it follows that

$$
\begin{aligned}
T_{(\Sigma, \mathbf{M}, \rho, \sigma)} \mathbf{E}= & \left\{(\delta \Sigma, \delta \mathbf{M}, \delta \rho, \delta \sigma) \mid \delta \Sigma \text { a function on } D_{\Sigma}, \delta \mathbf{M} \text { a vector field on } D_{\Sigma},\right. \\
& \delta \rho, \delta \sigma \text { functions on } D_{\Sigma} \text { such that there is a vector field } \mathbf{u} \text { on } D_{\Sigma} \\
& \text { satisfying: } \delta \Sigma=\mathbf{u} \cdot \nu, \delta \rho=-\operatorname{div}(\rho \mathbf{u}), \delta \sigma=-\mathbf{u} \cdot \nabla \sigma\}
\end{aligned}
$$

By (21), (22), (25), (26) and the change of variables $x=\eta(X)$ which provides the connection between the Lagrangian and the Eulerian representations, we have

$$
\begin{aligned}
\mathbf{D}_{\eta} F^{\prime}(\eta, \mu) \cdot \delta \eta= & \left.\frac{\mathrm{d}}{\mathrm{d} \varepsilon}\right|_{\varepsilon=0} F^{\prime}\left(\eta_{\varepsilon}, \mu_{\varepsilon}\right)= \\
= & \int_{\Sigma} \frac{\delta F}{\delta \Sigma} \delta \Sigma \mathrm{d} A+\int_{D_{\Sigma}} \frac{\delta F}{\delta \mathbf{M}} \cdot \delta \mathbf{M} \mathrm{d}^{3} \mathbf{x}+ \\
& +\int_{D_{\Sigma}} \frac{\delta F}{\delta \rho} \cdot \delta \rho \mathbf{d}^{3} \mathbf{x}+\int_{D_{\Sigma}} \frac{\delta F}{\delta \sigma} \cdot \delta \sigma \mathrm{d}^{3} \mathbf{x}= \\
= & \int_{\Sigma} \frac{\delta F}{\delta \Sigma}(\mathbf{x}) \delta \eta(\mathbf{X}) \cdot \nu(\mathbf{x}) \mathrm{d} A- \\
& -\int_{D_{\Sigma}} \frac{\delta F}{\delta \mathbf{M}}(\mathbf{x}) \cdot\left[\mathbf{M}(\mathbf{x}) \operatorname{div}\left(\delta \eta \circ \eta^{-1}\right)(\mathbf{x})+\right. \\
& \left.+\left(\left(\delta \eta \circ \eta^{-1}\right)(\mathbf{x}) \cdot \nabla\right) \mathbf{M}(\mathbf{x})\right] \mathrm{d}^{3} \mathbf{x}- \\
& -\int_{D_{\Sigma}} \mathrm{div}\left(\rho\left(\delta \eta \circ \eta^{-1}\right)\right)(\mathbf{x}) \mathrm{d}^{3} \mathbf{x}- \\
& -\int_{D_{\Sigma}} \frac{\delta F}{\delta \rho} \mathrm{div}\left(\rho\left(\delta \eta \circ \eta^{-1}\right)\right)(\mathbf{x}) \mathrm{d}^{3} \mathbf{x}- \\
& -\int_{D_{\Sigma}} \frac{\delta F}{\delta \sigma}(\mathbf{x}) \nabla \sigma(\mathbf{x}) \cdot \delta \eta(\mathbf{X}) \mathrm{d}^{3} \mathbf{x}= \\
= & \int_{\partial D}\left(\frac{\delta F}{\delta \Sigma} \circ \eta\right)(\mathbf{X}) \cdot \delta \eta(\mathbf{X}) j(\eta) \mathrm{d} a- \\
& -\int_{\Sigma} \frac{\delta F}{\delta \mathbf{M}}(\mathbf{x}) \cdot \mathbf{M}(\mathbf{x}) \delta \eta(\mathbf{X}) \cdot \nu(\mathbf{x}) \mathrm{d} A+ \\
& +\int_{D_{\Sigma}} \nabla\left(\frac{\delta F}{\delta \mathbf{M}}(\mathbf{x}) \cdot \mathbf{M}(\mathbf{x})\right) \delta \eta(\mathbf{X}) \mathrm{d}^{3} \mathbf{x}- \\
& -\int_{D_{\Sigma}} \delta \eta(\mathbf{X}) \cdot \frac{\delta F}{\delta \mathbf{M}}(\mathbf{x}) \nabla \mathbf{M}{ }^{i}(\mathbf{x}) \mathrm{d}^{3} \mathbf{x}- \\
& -\int_{\Sigma} \frac{\delta F}{\delta \rho}(\mathbf{x}) \rho(\mathbf{x}) \delta \eta(\mathbf{X}) \cdot \nu(\mathbf{x}) \mathrm{d} A+ \\
& \\
&
\end{aligned}
$$




$$
\begin{aligned}
& +\int_{D_{\mathbf{\Sigma}}} \rho(\mathbf{x}) \delta \eta(\mathbf{X}) \cdot \nabla \frac{\delta F}{\delta \rho}(\mathbf{x}) \mathbf{d}^{3} \mathbf{x}- \\
& -\int_{D}\left(\frac{\delta F}{\delta \sigma} \circ \eta\right)(\mathbf{X}) \cdot(\nabla \sigma \circ \eta)(\mathbf{X}) J(\eta)(\mathbf{X}) \mathrm{d}^{3} \mathbf{X}= \\
& =\int_{\partial D} j(\eta)(\mathbf{X})\left(\left[\left(\frac{\delta F}{\delta \Sigma}-\frac{\delta F}{\delta \mathbf{M}} \cdot \mathbf{M}-\frac{\delta F}{\delta \rho} \rho\right) \nu\right] \circ \eta\right)(\mathbf{X}) \cdot \delta \eta(\mathbf{X}) \mathrm{d} a+ \\
& +\int_{D} J(\eta)(\mathbf{X})\left(\left(\nabla\left(\frac{\delta F}{\delta \mathbf{M}} \cdot \mathbf{M}\right)-\right.\right. \\
& \left.\left.-\frac{\delta F}{\delta \mathbf{M}^{i}} \nabla \mathbf{M}^{i}+\rho \nabla \frac{\delta F}{\delta \rho}-\frac{\delta F}{\delta \sigma} \nabla \sigma\right) \circ \eta\right)(\mathbf{X}) \cdot \delta \eta(\mathbf{X}) \mathrm{d}^{3} \mathbf{X}
\end{aligned}
$$

which says by (10) that

$$
\begin{aligned}
& \frac{\delta^{\wedge} F^{\prime}}{\delta \eta}=J(\eta)\left[\left(\mathbf{M}^{i} \nabla \frac{\delta F}{\delta \mathbf{M}^{i}}+\rho \nabla \frac{\delta F}{\delta \rho}-\frac{\delta F}{\delta \sigma} \nabla \sigma\right) \circ \eta\right] \mathbf{d}^{3} \mathbf{X} \\
& \frac{\delta^{\sim} F^{\prime}}{\delta \eta}=j(\eta)\left[\left(\left(\frac{\delta F}{\delta \Sigma}-\frac{\delta F}{\delta \mathbf{M}} \cdot \mathbf{M}-\frac{\delta F}{\delta \rho} \rho\right) \nu\right) \circ \eta\right] \mathrm{d} a,
\end{aligned}
$$

where $\nu$ is the outward unit normal to $\partial D$.

Condition (13) is trivially verified by the second relation (20), which also shows that the second integral in (14) vanishes. Plugging in (20), (27), (28) into (14) and changing variables via $\mathbf{x}=\eta(\mathbf{X})$ results in the Poisson bracket on $\mathbf{E}$ :

$$
\begin{aligned}
\{F, K\}(\Sigma, \mathbf{M}, \rho, \sigma) & =\int_{D_{\Sigma}} \mathbf{M} \cdot\left[\left(\frac{\delta K}{\delta \mathbf{M}} \cdot \nabla\right) \frac{\delta F}{\delta \mathbf{M}}-\left(\frac{\delta F}{\delta \mathbf{M}} \cdot \nabla\right) \frac{\delta K}{\delta \mathbf{M}}\right] \mathrm{d}^{3} \mathbf{x}+ \\
& +\int_{D_{\Sigma}}\left(\frac{\delta K}{\delta \rho} \operatorname{div}\left(\rho \frac{\delta F}{\delta \mathbf{M}}\right)-\frac{\delta F}{\delta \rho} \operatorname{div}\left(\rho \frac{\delta K}{\delta \mathbf{M}}\right)\right) \mathrm{d}^{3} \mathbf{x}+ \\
& \left.+\int_{D_{\Sigma}} \nabla \sigma \cdot\left(\frac{\delta K}{\delta \sigma} \frac{\delta F}{\delta \mathbf{M}}-\frac{\delta F}{\delta \sigma} \frac{\delta K}{\delta \mathbf{M}}\right)\right) \mathrm{d}^{3} \mathbf{x}+ \\
& +\int_{\Sigma}\left[\left(\mathbf{M} \cdot \frac{\delta K}{\delta \mathbf{M}}\right) \frac{\delta F}{\delta \mathbf{M}}-\left(\mathbf{M} \cdot \frac{\delta F}{\delta \mathbf{M}}\right) \frac{\delta K}{\delta \mathbf{M}}\right] \cdot \nu \mathrm{d} A+ \\
& +\int_{\Sigma}\left(\frac{\delta F}{\delta \Sigma} \frac{\delta K}{\delta \mathbf{M}}-\frac{\delta K}{\delta \Sigma} \frac{\delta F}{\delta \mathbf{M}}\right) \cdot \nu \mathrm{d} A
\end{aligned}
$$

Note that the bracket (29) makes perfect sense on the union $\mathbf{E}^{\prime}=\{(\Sigma, \mathbf{M}, \rho, \sigma) \mid \rho=$ $\left(\rho_{0} \circ \phi^{-1}\right)\left(J(\phi)^{-1} \circ \phi^{-1}\right), \sigma=\sigma_{0} \circ \phi^{-1}$, for some $\rho_{0}, \sigma_{0}$, and $\left.\phi \in \mathbf{C}\right\}$ over all $\left(\rho_{0}, \sigma_{0}\right)$ of the manifolds $\mathbf{E}$ and that each $\mathbf{E}$ becomes thus a Poisson submanifold of $\mathbf{E}^{\prime}$. Moreover, using formulas (35), (37), and (38) developed in the next section, it is easily checked that the total mass and the total entropy are Casimirs on $\mathbf{E}^{\prime}$; their common level sets are the manifolds $\mathbf{E}$. 
Remark. There is another, geometrically more natural description of the Poisson bracket involving connections on semidirect product bundles; see Montgomery et al. [12] for the abstract theory and Lewis et al. [1] for the incompressible case. Also, (29) could be deduced from the work of Abarbanel and Holm [16] by specializing their result to Lagrangian variables on a surface.

\section{COMPUTATION OF FUNCTIONAL DERIVATIVES}

We will compute the functional derivatives on $\mathbf{E}$ of functions of the form

$$
F(\Sigma, \mathbf{M}, \rho, \sigma)=\int_{D_{\Sigma}} f(\mathbf{M}, \rho, \sigma) \mathrm{d}^{3} \mathbf{x}+\int_{\Sigma} g(\mathbf{x}) \mathrm{d} A
$$

for $C^{1}$ functions $f: \mathbb{R}^{3} \times \mathbb{R} \times \mathbb{R} \rightarrow \mathbb{R}, g: \mathbb{R}^{3} \rightarrow \mathbb{R}$. The definition of the functional derivatives, (15), is used. The Eulerian perturbation is obtained by perturbing th Lagrangian variables and projecting this perturbation onto $T \mathbf{E}$ via the tangent map of the projection map $\Pi$.

To carry out this approach, it first necessary to recall two formulas

$$
\left.\frac{\mathrm{d}}{\mathrm{d} \varepsilon}\right|_{\varepsilon=0} \int_{\eta_{\varepsilon}\left(D_{\Sigma}\right)} f(\mathbf{x}, \varepsilon) \mathrm{d}^{3} \mathbf{x}=\int_{\eta_{0}\left(D_{\Sigma}\right)}\left(\left.\frac{\partial f}{\partial \varepsilon}\right|_{\varepsilon=0}+\operatorname{div}(f \mathbf{u})\right) \mathrm{d}^{3} \mathbf{x}
$$

and

$$
\left.\frac{\mathrm{d}}{\mathrm{d} \varepsilon}\right|_{\varepsilon=0} \int_{\eta_{\varepsilon}(\Sigma)} g(\mathbf{x}) \mathrm{d} A=\int_{\eta_{0}(\Sigma)}\left(\frac{\partial g}{\partial \nu}+g \kappa\right) \delta \Sigma \mathrm{d} A
$$

where in both formulas, $\eta_{\varepsilon}$ is a flow over $D_{\Sigma}$ and $\mathbf{u}=\mathrm{d} \eta_{\varepsilon} /\left.\mathrm{d} \varepsilon\right|_{\varepsilon=0} \cdot \operatorname{In}(32), \delta \Sigma=\mathbf{u} \cdot \nu$ and $\kappa$ is the mean curvature of $\Sigma$. Formula (31) is the transport theorem and (32) is an analogue of the transport theorem restricted to the boundary of the domain (see e.g., Lawson [1977]).

In what follows, we identify the Lagrangian and Eulerian variables when $\varepsilon=0$ so that $\eta_{0}(\mathbf{X})=\mathbf{x}, \mu\left(\eta_{0}(\mathbf{X})\right)=\mathbf{M}(\mathbf{x}), \rho_{0}(\mathbf{X})=\rho(\mathbf{x}), \sigma_{0}(\mathbf{X})=\sigma(\mathbf{x})$ and $\Sigma=\partial D$. We also denote the point $\left(\left(\eta_{0}, \mu_{0}\right) \times\left(\rho_{0}, \sigma_{0}\right)\right) \in T^{*} \mathbf{C}$ by $L$.

From (31), (32) and the divergence theorem we find

$$
\begin{aligned}
\mathbf{D} F(\Sigma, \mathbf{M}, \rho, \sigma) & \cdot(\delta \Sigma, \delta \mathbf{M}, \delta \rho, \delta \sigma)=\mathbf{D} F(\Sigma, \mathbf{M}, \rho, \sigma) \cdot T_{L} \Pi(\delta \eta, \delta \mu)= \\
= & \mathbf{D} F(\Sigma, \mathbf{M}, \rho, \sigma) \cdot\left(T_{L} \Pi(\delta \eta, 0)+T_{L} \Pi(0, \delta \mu)\right)= \\
= & \left.\frac{\mathrm{d}}{\mathrm{d} \varepsilon}\right|_{\varepsilon=0} \int_{\eta_{\varepsilon}\left(D_{\Sigma}\right)} f(\mathbf{M}(\mathbf{x}, \varepsilon), \rho(\mathbf{x}, \varepsilon), \sigma(\mathbf{x}, \varepsilon)) \mathbf{d}^{3} \mathbf{x}+ \\
& +\left.\frac{\mathrm{d}}{\mathrm{d} \varepsilon}\right|_{\varepsilon=0} \int_{\eta_{\varepsilon}(\Sigma)} g(\mathbf{x}) \mathrm{d} A+ \\
& +\left.\frac{\mathrm{d}}{\mathrm{d} \varepsilon}\right|_{\varepsilon=0} \int_{D_{\Sigma}} \frac{\partial f}{\partial \mathbf{M}} \cdot T_{L} \Pi(0, \delta \mu) \mathrm{d}^{3} \mathbf{X}=
\end{aligned}
$$




$$
\begin{aligned}
= & \int_{D_{\Sigma}}\left[\frac{\partial f}{\partial \mathbf{M}}\left(\left.\frac{\partial \mathbf{M}}{\partial \varepsilon}\right|_{\varepsilon=0}+T_{L} \Pi(0, \delta \mu)\right)+\right. \\
& \left.+\left.\frac{\partial f}{\partial \rho} \frac{\partial \rho}{\partial \varepsilon}\right|_{\varepsilon=0}+\left.\frac{\partial f}{\partial \sigma} \frac{\partial \sigma}{\partial \varepsilon}\right|_{\varepsilon=0}\right] \mathrm{d}^{3} \mathbf{x}+ \\
& +\int_{\Sigma}\left(f+\frac{\partial g}{\partial \nu}+g \kappa\right)\left(\left(\delta \eta \circ \eta_{0}^{-1}\right) \cdot \nu\right) \mathrm{d} A= \\
= & \int_{D_{\Sigma}}\left(\frac{\partial f}{\partial \mathbf{M}} \cdot \delta \mathbf{M}+\frac{\partial f}{\partial \rho} \cdot \delta \rho+\frac{\partial f}{\partial \sigma} \cdot \delta \sigma\right) \mathrm{d}^{3} \mathbf{x}+ \\
& +\int_{\Sigma}\left(f+\frac{\partial g}{\partial \nu}+g \kappa\right) \mathrm{d} \Sigma \mathrm{d} A
\end{aligned}
$$

where $\delta \Sigma, \delta \sigma, \delta \rho$ are given by (21), (22), and $\delta \mathbf{M}=(26)+\delta \mu \circ \eta_{0}^{-1} ;$ in addition, $\eta_{\varepsilon}$ is a curve in the space of configurations $\mathbf{C}$ such that

$$
\left.\frac{\mathrm{d}}{\mathrm{d} \varepsilon}\right|_{\varepsilon=0} \eta_{\varepsilon}=\delta \eta
$$

The definition (15) along with (33) give

$$
\begin{aligned}
& \frac{\delta F}{\delta \Sigma}=f(\mathbf{M}, \rho, \sigma) \mid \Sigma+\frac{\partial g}{\partial \nu}+g \kappa \\
& \frac{\delta F}{\delta \mathbf{M}}=\frac{\partial f}{\partial \mathbf{M}} \\
& \frac{\delta F}{\delta \rho}=\frac{\partial f}{\partial \rho} \\
& \frac{\delta F}{\delta \rho}=\frac{\partial f}{\partial \rho} .
\end{aligned}
$$

In the next section we will need the functional derivatives of a gravitational energy of the form

$$
K(\Sigma, \rho)=-\frac{1}{2} \int_{D_{\Sigma}} \int_{D_{\Sigma}} \frac{\rho(\mathbf{x}) \rho(\mathbf{y})}{\|\mathbf{x}-\mathbf{y}\|} \mathrm{d}^{3} \mathbf{y} \mathrm{d}^{3} \mathbf{x}
$$

$K$ is the independent of $\mathbf{M}$ and $\sigma$, so that $\frac{\delta K}{\delta \mathbf{M}}=0, \frac{\delta K}{\delta \sigma}=0$. Since the variables $(\Sigma, \rho)$ are independent of the Lagrangian variable $\mu$, we need only perturb the Lagrangian variable $\eta$ and apply the transport theorem with mass density to the double integral (39). The transport theorem with mass density states

$$
\begin{aligned}
& \left.\frac{\mathrm{d}}{\mathrm{d} \varepsilon}\right|_{\varepsilon=0} \int_{\eta_{\varepsilon}\left(D_{\Sigma}\right)} \rho(\mathbf{x}, \varepsilon) f(\mathbf{x}) \mathrm{d}^{3} \mathbf{x}= \\
& =\int_{D_{\Sigma}} \rho(\mathbf{x}) \nabla f(\mathbf{x}) \cdot\left(\delta \eta \circ \eta_{0}^{-1}\right)(\mathbf{x}) \mathrm{d}^{3} \mathbf{x} .
\end{aligned}
$$


Let

$$
U(\mathbf{x})=\int_{D_{\Sigma}} \frac{\rho(\mathbf{y})}{\|\mathbf{x}-\mathbf{y}\|} \mathrm{d}^{3} \mathbf{y}
$$

Then using (21), (25), (39), (40) and the divergence theorem, we have (42)

$\mathbf{D} K(\Sigma, \rho) \cdot(\delta \rho, \delta \Sigma)=\mathbf{D} K(\Sigma, \rho) \cdot T_{L} \Pi(\delta \eta, 0)=$

$$
\begin{aligned}
= & -\left.\frac{1}{2} \frac{\mathrm{d}}{\mathrm{d} \varepsilon}\right|_{\varepsilon=0} \int_{\eta_{\varepsilon}\left(D_{\Sigma}\right)} \int_{\eta_{\varepsilon}\left(D_{\Sigma}\right)} \frac{\rho(\mathbf{x}) \rho(\mathbf{y})}{\|\mathbf{x}-\mathbf{y}\|} \mathrm{d}^{3} \mathbf{y} \mathrm{d}^{3} \mathbf{x}= \\
= & -\frac{1}{2} \int_{D_{\Sigma}} \rho(\mathbf{x})\left(\nabla U(\mathbf{x}) \cdot\left(\delta \eta \circ \eta_{0}^{-1}\right)(\mathbf{x})+\right. \\
& \left.+\left.\frac{\mathrm{d}}{\mathrm{d} \varepsilon}\right|_{\varepsilon=0} \int_{\eta_{\varepsilon}\left(D_{\Sigma}\right)} \frac{\rho(\mathbf{y})}{\|\mathbf{x}-\mathbf{y}\|} \mathrm{d}^{3} \mathbf{y}\right) \mathrm{d}^{3} \mathbf{x}= \\
= & -\frac{1}{2} \int_{D_{\Sigma}}\left[\rho(\mathbf{x}) \nabla_{\mathbf{x}} U(\mathbf{x}) \cdot\left(\delta \eta \circ \eta_{0}^{-1}\right)(\mathbf{x})+\right. \\
& \left.+\rho(\mathbf{y}) \nabla_{\mathbf{y}}\left(\int_{D_{\Sigma}} \frac{\rho(\mathbf{x})}{\|\mathbf{x}-\mathbf{y}\|} \mathrm{d}^{3} \mathbf{y}\right) \cdot\left(\delta \eta \circ \eta_{0}^{-1}\right)(\mathbf{y})\right] \mathrm{d}^{3} \mathbf{x}= \\
= & -\int_{D_{\Sigma}} \rho(\mathbf{x}) \nabla U(\mathbf{x}) \cdot\left(\delta \eta_{0} \circ \eta_{0}^{-1}\right)(\mathbf{x}) \mathrm{d}^{3} \mathbf{x}= \\
= & -\int_{D_{\Sigma}} U \mathrm{div}\left(-\rho\left(\delta \eta \circ \eta_{0}^{-1}\right)\right) \mathrm{d}^{3} \mathbf{x}- \\
& -\int_{\Sigma} \rho U\left(\left(\delta \eta \circ \eta_{0}^{-1}\right) \cdot \nu\right) \mathrm{d} A= \\
= & -\int_{D_{\Sigma}} U \delta \rho \mathrm{d}^{3} \mathbf{x}-\int_{\Sigma} \rho U \delta \Sigma \mathrm{d} A .
\end{aligned}
$$

Therefore, from (15),

$$
\begin{aligned}
& \frac{\delta K}{\delta \Sigma}=-\rho U \\
& \frac{\delta K}{\delta \rho}=-U
\end{aligned}
$$

Remark. To find the Casimir functionals on $\mathbf{E}$ (i.e., conserved quantities $C: \mathbf{E} \rightarrow \mathbb{R}$ such that $\{F, C\}=0$ for all $F: \mathbf{E} \rightarrow \mathbb{R}$ ), one should generalize th bracket (29) for a larger class of functionals admitting more general functional derivatives as in Lewis et al. [1]. However, for our case we need to allow for even more general functionals, which would complicate the bracket considerably. Instead we shall just limit ourselves 
to point out two classes of conserved quantities for the Hamiltonian (51). Let $\psi(\mathbf{x}, t)$ be any quantity advected by the flow, i.e., satisfying

$$
\frac{\partial \psi}{\partial t}+\mathbf{v} \cdot \nabla \psi=0
$$

Then denoting by $\omega=\operatorname{curl} \mathbf{v}$ the vorticity,

$$
\Psi=\frac{1}{\rho} \omega \cdot \nabla \psi
$$

is also advected, as an easy verification shows; see Ertel [18] and Pedlosky [19] sec. 25. In addition

$$
C(\Sigma, \mathbf{M}, \rho, \sigma)=\int_{D_{\Sigma}} \rho \Phi(\sigma, \Psi) \mathrm{d}^{3} \mathbf{x}
$$

for any $C^{1}$-function $\Psi: \mathbb{R}^{2} \rightarrow \mathbb{R}$, is a conserved quantity as an easy application of the transport theorem shows:

$$
\begin{aligned}
\frac{\mathrm{d}}{\mathrm{d} t} C & =\int_{D_{\Sigma}} \rho\left(\frac{\partial \Phi}{\partial t}+\mathbf{v} \cdot \nabla \Phi\right) \mathbf{d}^{3} \mathbf{x}= \\
& =\int_{D_{\Sigma}} \rho\left[\Phi_{\sigma}\left(\frac{\partial \sigma}{\partial t}+\mathbf{v} \cdot \nabla \sigma\right)+\right. \\
& \left.+\Phi_{\Psi}\left(\frac{\partial \Psi}{\partial t}+\mathbf{v} \cdot \nabla \Psi\right)\right] \mathrm{d}^{3} \mathbf{x}=0
\end{aligned}
$$

In particular, if $\Psi=\sigma$, then we get the potential vorticity $\Omega$ and if $\psi=X^{A}$, a Lagrange coordinate for the flow, we get the conserved quantities used in the fixed boundary case by Abarbancl and Holm [16].

\section{THE ADIABATIC SELF-GRAVITING LIQUID DROP WITH SURFACE TENSION}

Now we are going to turn to the physical problem of a sclf-gravitating inviscid fluid mass. The Hamiltonian formulation of this problem rests on the Poisson bracket we have just described. So we consider an adiabatic fluid with free boundary $\Sigma$ enclosing a compact domain $D_{\Sigma} \subset \mathbb{R}^{3}$ evolving under the influence of the potential density of self-gravitating

$$
\int_{D_{\Sigma}} \frac{\rho\left(\mathbf{x}^{\prime}\right)}{\left\|\mathbf{x}-\mathbf{x}^{\prime}\right\|} \mathrm{d}^{3} \mathbf{x}^{\prime}
$$


where $\rho$ is the Eulerian mass density of the fluid. We also assume that the fluid has constant surface tension $\tau$. The following notation will be used for the Eulerian description of the fluid:

- $\mathbf{v}$ is the Eulerian velocity;

- $\mathbf{M}=\rho \mathbf{v}$ is the Eulerian momentum density;

- $\sigma$ is the entropy;

$-\nu$ is the outward unit normal to $\Sigma$;

- $e(\rho, \sigma)$ is the internal inergy density of the fluid;

$-p(\mathbf{x})=\rho^{2}(\mathbf{x})(\partial e / \partial \rho)(\mathbf{x})$ is the pressure.

The equations of motion are:

- conservation of momentum

$$
\frac{\partial \mathbf{M}}{\partial t}=-(\mathbf{M} \cdot \nabla) \frac{\mathbf{M}}{\rho}-\frac{\mathbf{M}}{\rho} \operatorname{div} \mathbf{M}-\nabla p-\rho \nabla U
$$

- conservation of mass

$$
\frac{\partial \rho}{\partial t}=-\operatorname{div} \mathbf{M}
$$

- conservation of entropy (which is our definition of an adiabatic fluid)

$$
\frac{\partial \sigma}{\partial t}=-\frac{\mathbf{M}}{\rho} \cdot \nabla \sigma
$$

- and boundary movement

$$
\frac{\partial \Sigma}{\partial t}=\frac{\mathbf{M}}{\rho} \cdot \nu
$$

The dynamical boundary condition at $\Sigma$ is

$$
p \mid \Sigma=\tau \kappa,
$$

where $\kappa$ is the mean curvature of $\Sigma$. The total energy of this system is given by

$$
\begin{aligned}
H(\Sigma, \mathbf{M}, \rho, \sigma) & =\int_{D_{\Sigma}} \frac{\|\mathbf{M}\|^{2}}{2 \rho} \mathrm{d}^{3} \mathbf{x}+\int_{D_{\Sigma}} \rho e(\rho, \sigma) \mathrm{d}^{3} \mathbf{x}+ \\
& +(1 / 2) \int_{D_{\Sigma}} \rho U \mathrm{~d}^{3} \mathbf{x}+\tau \int_{\Sigma} \mathrm{d} A .
\end{aligned}
$$

The first term in (51) represents the kinetic energy of the fluid, whereas the second is the standard potential energy of an adiabatic fluid. The third term is the potential energy arising from self-gravitation and the fourth is the potential energy associated with surface tension. 
PROPOSITION. The equations of motion (46)-(50) are equivalent to $\dot{F}=\{F, H\}$ for all functions $F$ possessing functional derivatives, where $H$ is given by (15).

Proof. Using (35)-(39), (43) and (44) the functional derivatives of $H$ are,

$$
\begin{aligned}
& \frac{\delta H}{\delta \mathbf{M}}=\mathbf{M} / \rho, \\
& \frac{\delta H}{\delta \rho}=-\frac{\|\mathbf{M}\|^{2}}{2 \rho^{2}}+e(\rho, \sigma)+\rho \frac{\partial e}{\partial \rho}+U, \\
& \frac{\delta H}{\delta \sigma}=\rho \frac{\partial e}{\partial \sigma}, \\
& \frac{\delta H}{\delta \Sigma}=\frac{\|\mathbf{M}\|^{2}}{2 \rho}+\rho e(\rho, \sigma)+\rho U+\tau \kappa .
\end{aligned}
$$

Therefore,

$$
\begin{aligned}
\{F, H\}= & \int_{D_{\Sigma}}\left[\mathbf{M} \cdot\left(\frac{\mathbf{M}}{\rho} \cdot \nabla\right) \frac{\delta F}{\delta \mathbf{M}}-\mathbf{M} \cdot\left(\frac{\delta F}{\delta \mathbf{M}} \cdot \nabla\right) \frac{\mathbf{M}}{\rho}-\frac{\delta F}{\delta \rho} \operatorname{div} \mathbf{M}-\right. \\
& -\rho \nabla\left(-\frac{\|\mathbf{M}\|^{2}}{2 \rho^{2}}+e(\rho, \sigma)+\rho \frac{\partial e}{\partial \rho}+U\right) \cdot \frac{\partial F}{\partial \mathbf{M}}+ \\
& \left.+\rho \frac{\partial e}{\partial \sigma} \nabla \sigma \cdot \frac{\delta F}{\delta \mathbf{M}}-\frac{\delta F}{\delta \sigma} \frac{\mathbf{M}}{\rho} \cdot \nabla \sigma\right] \mathrm{d}^{3} \mathbf{x}+ \\
& +\int_{\Sigma}\left[\frac{\|\mathbf{M}\|^{2}}{\rho} \frac{\delta F}{\delta \mathbf{M}}-\left(\mathbf{M} \cdot \frac{\delta F}{\delta \mathbf{M}}\right) \frac{\mathbf{M}}{\rho}+\right. \\
& +\left(-\frac{\|\mathbf{M}\|^{2}}{2 \rho}+\rho e(\rho, \sigma)+\rho^{2} \frac{\partial e}{\partial \rho}+\rho U\right) \frac{\delta F}{\delta \mathbf{M}}+\frac{\delta F}{\delta \Sigma} \frac{\mathbf{M}}{\rho}- \\
& \left.-\left(\frac{\|\mathbf{M}\|^{2}}{2 \rho}+\rho e(\rho, \sigma)+\rho U+\tau \kappa\right) \frac{\delta F}{\delta \mathbf{M}}\right] \cdot \nu \mathrm{d} A .
\end{aligned}
$$

The boundary term equals

$$
\int_{\Sigma}\left[-\left(\mathbf{M} \cdot \frac{\delta F}{\delta \mathbf{M}}\right) \frac{\mathbf{M}}{\rho}+\frac{\delta F}{\delta \Sigma} \frac{\mathbf{M}}{\rho}+(p-\tau \kappa) \frac{\delta F}{\delta \mathbf{M}}\right] \cdot \nu \mathrm{d} A
$$

Therefore we get

$$
\begin{aligned}
\{F, H\}= & \int_{D_{\Sigma}}\left[\mathbf{M} \cdot\left(\frac{\mathbf{M}}{\rho} \cdot \nabla\right) \frac{\delta F}{\delta \mathbf{M}}-\mathbf{M} \cdot\left(\frac{\delta F}{\delta \mathbf{M}} \cdot \nabla\right) \frac{\mathbf{M}}{\rho}-\right. \\
& -\rho \nabla\left(-\frac{\underline{\mathbf{M}}\rfloor^{2}}{2 \rho^{2}}+e(\rho, \sigma)+\rho \frac{\partial e}{\partial \rho}+U\right) \cdot \frac{\delta F}{\delta \mathbf{M}}+ \\
& \left.+\rho \frac{\partial e}{\partial \sigma} \nabla \sigma \cdot \frac{\delta F}{\delta \mathbf{M}}--\frac{\delta F}{\delta \rho} \operatorname{div} \mathbf{M} \frac{\delta F}{\delta \sigma} \frac{\mathbf{M}}{\rho} \cdot \nabla \sigma\right] \mathrm{d}^{3} \mathbf{x}+ \\
& +\int_{\Sigma}\left[-\left(\mathbf{M} \cdot \frac{\delta F}{\delta \mathbf{M}}\right) \frac{\mathbf{M}}{\rho}+\frac{\delta F}{\delta \Sigma} \frac{\mathbf{M}}{\rho}-(p-\tau \kappa) \frac{\delta F}{\delta \mathbf{M}}\right] \cdot \nu \mathrm{d} A .
\end{aligned}
$$


We need to isolate $\frac{\delta F}{\delta \mathbf{M}}$ in the volume integral. Since

$$
\rho \nabla\left(e(\rho, \sigma)+\rho \frac{\partial e}{\partial \rho}\right)=\nabla p+\rho \frac{\partial e}{\partial \sigma} \nabla \sigma
$$

by the definition of $p=\rho^{2} \partial e / \partial \rho$, the fourth term in the volume integral in (58) cancels.

Since

$$
\begin{aligned}
\left(\frac{\mathbf{M}}{\rho} \cdot \nabla\right) \frac{\delta F}{\delta \mathbf{M}} & =-\left(\frac{\delta F}{\delta \mathbf{M}} \cdot \nabla\right) \frac{\mathbf{M}}{\rho}-\frac{\mathbf{M}}{\rho} \times \operatorname{curl} \frac{\delta F}{\delta \mathbf{M}}- \\
& -\frac{\delta F}{\delta \mathbf{M}} \times \operatorname{curl} \frac{\mathbf{M}}{\rho}+\nabla\left(\frac{\mathbf{M}}{\rho} \cdot \frac{\delta F}{\delta \mathbf{M}}\right)
\end{aligned}
$$

and the second and third term below cancel, we have

$$
\begin{aligned}
\mathbf{M} & \left(\frac{\mathbf{M}}{\rho} \cdot \nabla\right) \frac{\delta F}{\delta \mathbf{M}}-\mathbf{M} \cdot\left(\frac{\delta F}{\delta \mathbf{M}} \cdot \nabla\right) \frac{\mathbf{M}}{\rho}+\rho \frac{\delta F}{\delta \mathbf{M}} \cdot \nabla\left(\frac{\|\mathbf{M}\|^{2}}{2 \rho^{2}}\right)= \\
= & -\mathbf{M} \cdot\left(\frac{\delta F}{\delta \mathbf{M}} \cdot \nabla\right) \frac{\mathbf{M}}{\rho}-\frac{\delta F}{\delta \mathbf{M}} \cdot\left(\operatorname{curl} \frac{\mathbf{M}}{\rho} \times \mathbf{M}\right)+ \\
& +\mathbf{M} \cdot \nabla\left(\frac{\mathbf{M}}{\rho} \cdot \frac{\delta F}{\delta \mathbf{M}}\right)= \\
= & -\rho \frac{\delta F}{\delta \mathbf{M}} \cdot\left[\nabla\left(\frac{\|\mathbf{M}\|^{2}}{2 \rho^{2}}\right)+\operatorname{curl} \frac{\mathbf{M}}{\rho} \times \frac{\mathbf{M}}{\rho}\right]+ \\
& +\operatorname{div}\left(\left(\mathbf{M} \cdot \frac{\delta F}{\delta \mathbf{M}}\right) \frac{\mathbf{M}}{\rho}\right)-\frac{\delta F}{\delta \mathbf{M}} \cdot \frac{\mathbf{M}}{\rho} \operatorname{div} \mathbf{M}= \\
= & -\frac{\delta F}{\delta \mathbf{M}} \cdot\left[(\mathbf{M} \cdot \nabla) \frac{\mathbf{M}}{\rho}+\frac{\mathbf{M}}{\rho} \operatorname{div} \mathbf{M}\right]+\operatorname{div}\left(\left(\mathbf{M} \cdot \frac{\delta F}{\delta \mathbf{M}}\right) \frac{\mathbf{M}}{\rho}\right),
\end{aligned}
$$

so that first four terms in the volume integral of (58) become

$$
\begin{aligned}
& \int_{D_{\Sigma}} \frac{\partial F}{\partial \mathbf{M}} \cdot\left[-(\mathbf{M} \cdot \nabla) \frac{\mathbf{M}}{\rho}-\frac{\mathbf{M}}{\rho} \operatorname{div} \mathbf{M}-\nabla p-\rho \nabla U\right] \mathrm{d}^{3} \mathbf{x}+ \\
& +\int_{\Sigma}\left(\mathbf{M} \cdot \frac{\delta F}{\delta \mathbf{M}}\right) \frac{\mathbf{M}}{\rho} \cdot \nu \mathrm{d} A .
\end{aligned}
$$

From (58) and (59) it then follows that

$$
\begin{aligned}
\{F, H\}= & \int_{D_{\Sigma}}\left[\frac{\delta F}{\delta \mathbf{M}} \cdot\left(-(\mathbf{M} \cdot \nabla) \frac{\mathbf{M}}{\rho}-\frac{\mathbf{M}}{\rho} \operatorname{div} \mathbf{M}-\nabla p-\rho \nabla U\right)-\right. \\
& \left.-\frac{\delta F}{\delta \rho} \operatorname{div} \mathbf{M}-\frac{\delta F}{\delta \rho} \frac{\mathbf{M}}{\rho} \cdot \nabla \sigma\right] \mathrm{d}^{3} \mathbf{x}+ \\
& +\int_{\Sigma}\left[\frac{\delta F}{\delta \Sigma} \frac{\mathbf{M}}{\rho}+(p-\tau \kappa) \frac{\delta F}{\delta \mathbf{M}}\right] \cdot \nu \mathrm{d} A .
\end{aligned}
$$


On the other hand,

$$
\begin{aligned}
\dot{F}= & \int_{D_{\Sigma}}\left(\frac{\delta F}{\delta \mathbf{M}} \cdot \frac{\partial \mathbf{M}}{\partial t}+\frac{\delta F}{\delta \rho} \cdot \frac{\partial \rho}{\partial t}+\frac{\delta F}{\delta \sigma} \cdot \frac{\partial \sigma}{\partial t}\right) \mathrm{d}^{3} \mathbf{x}+ \\
& +\int_{\Sigma} \frac{\delta F}{\delta \Sigma} \cdot \frac{\partial \Sigma}{\partial t} \mathrm{~d} A
\end{aligned}
$$

so that (60) and (61) coincide for any $F$ if and only if the equations of motion (46)-(49) and the boundary condition (50) hold.

\section{A POISSON MAP}

In Abarbanel et al. [11], the free boundary is considered as the zero set of a function $S$, i.e., $\Sigma=S^{-1}(0)$. Requiring that $S$ be advected by the flow, a formal application of the semidirect product theory (Ratiu [17], Marsden, Ratiu, Weinstein [10]) yields the following Poisson bracket

$$
\begin{aligned}
\left\{F^{\prime}, K^{\prime}\right\}^{\prime}= & \int \mathbf{M}^{\prime} \cdot\left[\left(\frac{\delta K^{\prime}}{\delta \mathbf{M}^{\prime}} \cdot \nabla\right) \frac{\delta F^{\prime}}{\delta \mathbf{M}^{\prime}}-\left(\frac{\delta F^{\prime}}{\delta \mathbf{M}^{\prime}} \cdot \nabla\right) \frac{\delta K^{\prime}}{\delta \mathbf{M}^{\prime}}\right] \mathrm{d}^{3} \mathbf{x}+ \\
& +\int \rho^{\prime}\left[\frac{\delta K^{\prime}}{\delta \mathbf{M}^{\prime}} \cdot \nabla \frac{\delta F^{\prime}}{\delta \rho^{\prime}}-\frac{\delta F^{\prime}}{\delta \mathbf{M}^{\prime}} \cdot \nabla \frac{\delta K^{\prime}}{\delta \rho^{\prime}}\right] \mathrm{d}^{3} \mathbf{x}+ \\
& +\int\left[\frac{\delta K^{\prime}}{\delta \sigma^{\prime}} \cdot \frac{\delta F^{\prime}}{\delta \mathbf{M}^{\prime}}-\frac{\delta F^{\prime}}{\delta \sigma^{\prime}} \cdot \frac{\delta K^{\prime}}{\delta \mathbf{M}^{\prime}}\right] \cdot \nabla \sigma^{\prime} \mathbf{d}^{3} \mathbf{x}+ \\
& +\int\left[\frac{\delta K^{\prime}}{\delta S} \cdot \frac{\delta F^{\prime}}{\delta \mathbf{M}^{\prime}}-\frac{\delta F^{\prime}}{\delta S} \cdot \frac{\delta K^{\prime}}{\delta \mathbf{M}^{\prime}}\right] \cdot \nabla S \mathbf{d}^{3} \mathbf{x}+
\end{aligned}
$$

where all integrals are taken over $\mathbf{R}^{3}$ and one thinks of $\mathbf{R}^{3}$ as filled with two species of fluid, one inside $(S<0)$ and one outside $(S>0)$ of the boundary $\Sigma=S^{-1}(0)$. In Abarbanel et al. [11] this bracket is obtained by reduction from the Lagrangian representation.

Consider the obvious restriction map $R:\left(S, \mathbf{M}^{\prime}, \rho^{\prime}, \sigma^{\prime}\right) \mapsto(\Sigma, \mathbf{M}, \rho, \sigma)$, where $\Sigma=S^{-1}(0), \mathbf{M}=\mathbf{M}^{\prime}\left|D_{\Sigma}, \rho=\rho^{\prime}\right| D_{\Sigma}, \sigma=\sigma^{\prime} \mid D_{\Sigma}$. We shall prove below that $R$ is a Poisson map (i.e., a map preserving the Poisson brackets), thus enabling one to pass freely from one formulation to the other and at the same time eliminating the redunancy introduced by $S$. That is, we shall prove that

$$
\{F \circ R, K \circ R\}^{\prime}=\{F, K\} \circ R
$$


for all functions $F, K: \mathbf{E} \rightarrow \mathbf{R}$. To evaluate the left hand side of (63) let us compute $\delta(F \circ R) / \delta \mathbf{M}^{\prime}$. If $\delta \mathbf{M}=\delta \mathbf{M}^{\prime} \mid D_{\Sigma}$ we get

$$
\begin{aligned}
\mathbf{D}_{\mathbf{M}}(F \circ R)\left(S, \mathbf{M}^{\prime}, \rho^{\prime}, \sigma^{\prime}\right) & =\int_{D_{\mathbf{\Sigma}}} \frac{\delta F}{\delta \mathbf{M}} \cdot \delta \mathbf{M} \mathrm{d}^{3} \mathbf{x}= \\
& =\int_{\mathbf{R}^{3}} \theta(-S) \frac{\delta F}{\delta \mathbf{M}} \cdot \delta \mathbf{M}^{\prime} \mathbf{d}^{3} \mathbf{x}
\end{aligned}
$$

where $\theta(-S)$ denotes the Heavyside function which is one on $D_{\Sigma}$ and zero outside of $D_{\Sigma}$. Therefore

$$
\frac{\delta(F \circ R)}{-\delta \mathbf{M}^{\prime}}=\theta(-S) \frac{\delta F}{\delta \mathbf{M}}
$$

and similarly

$$
\frac{\delta(F \circ R)}{\delta \rho^{\prime}}=\theta(-S) \frac{\delta F}{\delta \rho}, \quad \frac{\delta(F \circ R)}{\delta \sigma}=\theta(-S) \frac{\delta F}{\delta \sigma}
$$

Finally since the outward unit normal to $\Sigma$ is $\nu=\nabla S /\|\nabla S\|$, a perturbation $\delta S$ of $S$ induces a normal vector to the surface equal to $-(\delta S /\|\nabla S\|) \nu$, i.e., $\delta \Sigma=$ $-\delta S /\|\nabla S\|$. Thus

$$
\begin{aligned}
\int_{\mathbf{R}^{3}} \frac{\delta(F \circ R)}{\delta S} \delta S \mathrm{~d} \mathbf{x}^{3} & =\mathbf{D}_{S} F \cdot \delta S=\int_{\Sigma} \frac{\delta F}{\delta \Sigma} \delta \Sigma \mathrm{d} A= \\
& =-\int_{\Sigma} \frac{\delta F}{\delta \Sigma} \frac{\delta S}{\|\nabla S\|} \mathrm{d} A= \\
& =-\int_{\mathbf{R}^{3}} \frac{\delta F}{\delta \Sigma} \delta S \delta(\Sigma) \mathrm{d}^{3} \mathbf{x}
\end{aligned}
$$

since $\mathrm{d} A=\delta(\Sigma)\|\nabla S\| \mathrm{d}^{3} \mathbf{x}$ whence

$$
\frac{\delta(F \circ R)}{\delta S}=-\frac{\delta F}{\delta \Sigma} \delta(\Sigma)
$$

where $\delta(\Sigma)$ is the Dirac delta function of $\Sigma$. Denoting by $(F \leftarrow \rightarrow K)$ the expression in which $F$ and $K$ are interchanged, we get from (64), (65), (66): 


$$
\begin{aligned}
\{F \circ R, K \circ R\}^{\prime}= & \int_{\mathbf{R}^{3}} \mathbf{M}^{\prime} \theta(-S)\left(\frac{\delta K}{\delta \mathbf{M}} \cdot \nabla\right)\left(\theta(-S) \frac{\delta F}{\delta \mathbf{M}}\right) \mathrm{d}^{3} \mathbf{x}+ \\
& +\int_{\mathbf{R}^{3}} \rho^{\prime} \theta(-S) \frac{\delta K}{\delta \mathbf{M}} \cdot \nabla\left(\theta(-S) \frac{\delta F}{\delta \rho}\right) \mathrm{d}^{3} \mathbf{x}+ \\
& +\int_{\mathbf{R}^{3}} \theta(-S)^{2} \frac{\delta K}{\delta \sigma} \frac{\delta F}{\delta \mathbf{M}} \cdot \nabla \sigma^{\prime} \mathrm{d}^{3} \mathbf{x}- \\
& -\int_{\mathbf{R}^{3}} \theta(-S) \delta(\Sigma) \frac{\delta K}{\delta \Sigma} \frac{\delta F}{\delta \mathbf{M}} \cdot \nabla S \mathrm{~d}^{3} \mathbf{x}+(F \leftarrow \rightarrow K)= \\
& =\int_{D_{\Sigma}} \mathbf{M} \cdot\left(\frac{\delta K}{\delta \mathbf{M}} \cdot \nabla\right) \frac{\delta F}{\delta \mathbf{M}} \mathrm{d}^{3} \mathbf{x}- \\
& -\int_{\Sigma}\left(\mathbf{M} \cdot \frac{\delta F}{\delta \mathbf{M}}\right) \frac{\delta K}{\delta \mathbf{M}} \mathrm{d} A+\int_{D_{\Sigma}} \rho \frac{\delta K}{\delta \mathbf{M}} \cdot \nabla \frac{\delta F}{\delta \rho}- \\
& -\int_{\Sigma} \rho \frac{\delta K}{\delta \mathbf{M}} \frac{\delta F}{\delta \rho} \mathrm{d} A+\int_{D_{\Sigma}} \frac{\delta K}{\delta \sigma} \frac{\delta F}{\delta \mathbf{M}} \cdot \nabla \mathrm{d}^{3} \mathbf{x}- \\
& -\int_{\Sigma} \frac{\delta K}{\delta \Sigma} \frac{\delta F}{\delta \mathbf{M}} \cdot \nu \mathrm{d} A+(F \leftarrow \rightarrow K)
\end{aligned}
$$

which coincides with (29).

\section{ACKNOWLEDGEMENTS}

We would like to thank H.D.I. Abarbanel, R. Brown, D. Lewis, J. Marsden, A. Rouhi, and Y. Yang for many fruitful conversations during the work on this paper. In particular we appreciate the many useful editorial improvements provided by H.D.I. Abarbanel. T.R. was partially supported by NSF grant DMS 8701318-01 and AFOSR/DARPA contract F49620-87-C-0118.

\section{REFERENCES}

[1] D. Lewis, J. E. MARSDEN, R. MONTGOMERY, T. RATU: The Hamiltonian structure for dynamic free boundary problems, Physica 18D, 391, 1986.

[2] D. LEWIS, J. E. MARSDEN, T. RATIU: Stability and bifurcation of a rigidly rotating liquid drop, J. Math. Phys. 28, (10), 2508, 1987.

[3] D. LEwIS: Rotating liquid drops: Hamiltonian structure, stability and bifurcation, Thesis, Mathematics Department, U.C. Berkeley, 1987.

[4] V.E. ZAKHAROV: Stability of periodic waves of finite amplitude on the surface of a decp fluid, Zh. Prikl. Mekh. Fiz. 9, 86, (Engl. transl. J. Appl. Mech. Tech. Phys. 2, 190), 1968.

[5] L.J.F. BRoER: On the Hamiltonian theory of surface waves, Appl. Sci. Res. 30, 430, 1974.

[6] J.W. MLLEs: On Hamilton's principle for surface waves, J. Fluid Mech. 83, 153, 1977.

[7] D.M. MLDER: A note regarding "On Hamilton's principle for surface waves», J. Fluid Mech. 83, 159, 1977. 
[8] T.B. BENJAMIN, P.J. OLVER: Hamiltonian structure, symmetries and conservation laws for water waves, J. Fluid Mech. 125, 137, 1982.

T.B. BENJAMIN: Hamiltonian theory for motions of bubbles in an intuitive liquid, J. Fluid Mech. 181, 1987.

[9] F. HENYEY, J. WRIGHT : Hamiltonian description of the interaction of surface waves with mixed layer currents in Dynamics of the oceanic surface mixed layer, Proceedings, Hawaiian Winter Workshop, Jan. 14 - 16, 1987 (P. Muller, D. Henderson, eds.) Hawaii Institute of Geophysics Special Publication, 1987, 219 - 228.

[10] J.E. MARSDEN, T. RATIU, A. WEINSTEIN: Semidirect products and reduction in mechanics, Trans. Am. Math. Soc. 281, 147,1984 and Reduction and Hamiltonian structures on duals of semidirect product Lie algebras, Cont. Math. 28, 55, 1984.

[11] H.D.I. ABARBANEL, R. BROWN, Y.M. YANG: Hamiltonian formulation of inviscid flows with free boundaries, Phys. Fluids 31, 2802, 1988.

[12] R. Montgomery, J.E. Marsden, T. Ratiu: Gauged Lie-Poisson structures, Cont. Math. (AMS) 28, 101, 1984.

[13] H.D.I. AbARBANEl, D.D. Holm, J.E. MARSDEN, T. Ratiu: Nonlinear stability analysis of fuid equilibria, Phil. Trans. R. Soc. London A. 318, 349, 1987.

[14] D.D. HOLM, J.E. MARSDEN, T. RATIU, A. WEINSTEIN: Nonlinear stability of fluid and plasma equilibria, Phys. Rep. 1\&2, 1, 1985.

[15] H.B. LAWSON: Lectures on minimal surfaces, Publish or Perish 1977.

[16] H.D.I. ABARBANEL, D.D. HOLM: Nonlinear stability analysis of inviscid flows in three dimensions, Phys. Fluids 30, 3369, 1987.

[17] T. RATU: Euler-Poisson equations on Lie algebras and the $N$-dimensional heavy rigid body, Am. J. Math. 104, 409, 1337, 1982.

[18] H. ERTEL: Ein neuer hydrodynamisches Wirbelsatz, Meteorol. Z. 59, 277, 1942.

[19] J. PEDLOSKY: Geophysical Fluid Dynamics, Springer Study Edition, Springer 1982.

[20] D.D. HOLM, J.E. MARSDEN, T. RATTU: The Hamiltonian structure of continuum mechanics in material, inverse material, spatial, and convective representations, Séminaire de Mathématiques Superieur, Les Presses de l'Université de Montréal 1, 1986.

[21] H. POINCARE': Sur l'équilibre d'une masse fluide animée d'un mouvement de rotation, Acta Mathematica 7, 259, 1885.

Manuscript received: May 30, 1988 